\title{
A novel assistive method for rigidity evaluation during deep brain stimulation surgery using acceleration sensors
}

\author{
Ashesh Shah, MSc, ${ }^{1}$ Jérôme Coste, PhD, ${ }^{2,3}$ Jean-Jacques Lemaire, MD, PhD, ${ }^{2,3}$ \\ Erik Schkommodau, PhD, ${ }^{1}$ Ethan Taub, MD, ${ }^{4}$ Raphael Guzman, MD, ${ }^{4}$ Philippe Derost, MD,, 6 and \\ Simone Hemm, PhD ${ }^{1}$
}

${ }^{1}$ Institute for Medical and Analytical Technologies, School of Life Sciences, University of Applied Sciences and Arts Northwestern Switzerland, Muttenz; ${ }^{4}$ Departments of Neurosurgery and Biomedicine, University Hospital of Basel, Switzerland; ${ }^{2} I m a g e-G u i d e d$ Clinical Neuroscience and Connectomics, and ${ }^{5}$ Neuro-Psycho-Pharmacologie des Systèmes Dopaminergiques Sous-Corticaux, Université Clermont Auvergne, Université d'Auvergne; and ${ }^{3}$ Service de Neurochirurgie and ${ }^{6}$ Service de Neurologie, $\mathrm{CHU}$ Clermont-Ferrand, France

OBJECTIVE Despite the widespread use of deep brain stimulation (DBS) for movement disorders such as Parkinson's disease (PD), the exact anatomical target responsible for the therapeutic effect is still a subject of research. Intraoperative stimulation tests by experts consist of performing passive movements of the patient's arm or wrist while the amplitude of the stimulation current is increased. At each position, the amplitude that best alleviates rigidity is identified. Intrarater and interrater variations due to the subjective and semiquantitative nature of such evaluations have been reported. The aim of the present study was to evaluate the use of an acceleration sensor attached to the evaluator's wrist to assess the change in rigidity, hypothesizing that such a change will alter the speed of the passive movements. Furthermore, the combined analysis of such quantitative results with anatomy would generate a more reproducible description of the most effective stimulation sites.

METHODS To test the reliability of the method, it was applied during postoperative follow-up examinations of 3 patients. To study the feasibility of intraoperative use, it was used during 9 bilateral DBS operations in patients suffering from PD. Changes in rigidity were calculated by extracting relevant outcome measures from the accelerometer data. These values were used to identify rigidity-suppressing stimulation current amplitudes, which were statistically compared with the amplitudes identified by the neurologist. Positions for the chronic DBS lead implantation that would have been chosen based on the acceleration data were compared with clinical choices. The data were also analyzed with respect to the anatomical location of the stimulating electrode.

RESULTS Outcome measures extracted from the accelerometer data were reproducible for the same evaluator, thus providing a reliable assessment of rigidity changes during intraoperative stimulation tests. Of the 188 stimulation sites analyzed, the number of sites where rigidity-suppressing amplitudes were found increased from 144 to 170 when the accelerometer evaluations were considered. In general, rigidity release could be observed at significantly lower amplitudes with accelerometer evaluation (mean $0.9 \pm 0.6 \mathrm{~mA}$ ) than with subjective evaluation (mean $1.4 \pm 0.6 \mathrm{~mA})(p<0.001)$. Of 14 choices for the implant location of the DBS lead, only 2 were the same for acceleration-based and subjective evaluations. The comparison across anatomical locations showed that stimulation in the fields of Forel ameliorates rigidity at similar amplitudes as stimulation in the subthalamic nucleus, but with fewer side effects.

CONCLUSIONS This article describes and validates a new assistive method for assessing rigidity with acceleration sensors during intraoperative stimulation tests in DBS procedures. The initial results indicate that the proposed method may be a clinically useful aid for optimal DBS lead placement as well as a new tool in the ongoing scientific search for the optimal DBS target for PD.

https://thejns.org/doi/abs/10.3171/2016.8.JNS152770

KEY WORDS deep brain stimulation; Parkinson's disease; rigidity; acceleration sensor; quantification; intraoperative; subthalamic nucleus; fields of Forel; functional neurosurgery

\footnotetext{
ABBREVIATIONS AmpQ = quantitatively identified rigidity-suppressing amplitude; $\mathrm{AmpS}=$ subjectively assessed amplitude; $\mathrm{DBS}=$ deep brain stimulation; $\mathrm{FF}=$ fields of Forel; MER = microelectrode recording; OR = operating room; PD = Parkinson's disease; $Q \mathrm{C}=$ quantitatively assessed change; $\mathrm{S}$-EMG = surface electromyography; STN = subthalamic nucleus; USB = Universal Serial Bus; ZI = zona incerta.
}

SUBMITTED December 1, 2015. ACCEPTED August 24, 2016.

INCLUDE WHEN CITING Published online December 16, 2016; DOI: 10.3171/2016.8.JNS152770. 
$\mathrm{D}$ EEP brain stimulation (DBS) is a neurosurgical technique in which electrodes are implanted in deep-seated brain structures (e.g., the subthalamic nucleus [STN], globus pallidus, or thalamus) so that these structures can be stimulated with electrical pulses generated in an attached, extracranially implanted neurostimulator device. DBS is a highly effective symptomatic treatment for Parkinson's disease (PD) and other movement disorders. ${ }^{25}$ More than 100,000 patients have been treated with DBS in the past 3 decades. ${ }^{23}$

The current incomplete understanding of the mechanisms of action of DBS and interindividual variation of brain anatomy necessitate patient-specific planning. Most centers also perform intraoperative stimulation tests under local anesthesia at multiple anatomical locations in the vicinity of a preoperatively chosen target ${ }^{1}$ to evaluate the therapeutic effects and side effects of stimulation before the DBS lead (permanent electrode) is definitively fixed in place. For some patients with PD, the therapeutic effects of stimulation tests are estimated by assessing the changes in rigidity as the stimulation current is increased. Expert evaluators (generally a neurologist) perform passive movements of the patient's arm continuously to assess the changes in rigidity. When either a reduction in rigidity or a side effect is observed, the amplitude and the effect of stimulation are noted. After completion of all stimulation tests, the results are compared to identify the best implant position, that is, the one that yields the best improvement with the fewest side effects.

Different evaluators assess rigidity during surgery in different ways, ${ }^{5}$ but with the same basic concept: the baseline rigidity is rated before any stimulation, and the changes in rigidity are assessed in comparison with this baseline value. Intraoperatively used rating scales for PD are based on those commonly used outside the operating room (OR), mainly the Unified Parkinson's Disease Rating Scale ${ }^{7}$ and the Hoehn and Yahr staging scale..$^{10}$ Previous studies have shown that such rating scales have low interrater ${ }^{16,18}$ and intrarater ${ }^{18}$ reliability, because they are subjective and depend highly on the experience of the evaluating neurologist. ${ }^{8}$ Additionally, these scales have only discrete levels and were not designed for continuous rigidity evaluation. ${ }^{2}$ To increase the sensitivity for intraoperative use, some neurologists add intermediate levels to the existing rating scales..$^{12}$ An alternative way to increase sensitivity is to measure movement parameters during passive movement with sensors and thereby quantify the change in rigidity.

Various methods have been proposed for quantifying the absolute level of rigidity in patients with PD by measuring responses to passive movement. ${ }^{11,17,19}$ Some researchers have used surface electromyography (S-EMG) to measure muscle activity and have shown that the signals differ between healthy subjects and patients with PD..$^{15,26}$ Other techniques have also been proposed, which use a torque-motor setup to perform precise movements of a patient's arm or wrist while measuring joint angle and resistance. ${ }^{2,5,21}$ These studies showed that the features extracted from such precise movements are more strongly correlated with rating scales than those extracted from S-EMG signals. ${ }^{4}$ However, the intraoperative use of such techniques to quantify rigidity would be excessively cum- bersome or impossible. Usable signals from S-EMG electrodes can only be obtained when they are in close contact with the skin; the prolonged use of such electrodes might cause discomfort. In addition, the mechanical torque-motor setup limits the evaluation of rigidity to a single joint, and the apparatus is typically too large for use in the OR.

Rigidity is defined as the resistance to passive movement; thus, it can be presumed that during stimulation tests, the speed of the movement would increase with a reduction in rigidity. To the best of our knowledge, no such method has been proposed, in which the change in rigidity is assessed by measuring the change in speed (acceleration) of the passive movement. One reason for the lack of such a method could be the variations associated with the passive movements. In general, each expert evaluator has a preferred joint (arm/wrist) to perform passive movements to evaluate rigidity. Each expert can also choose the pattern of passive movement (extension, flexion, or circular) to be performed during a test. An expert may also stop and restart passive movements to prevent the patient's active participation in the movements. Therefore, any method for assessing rigidity that is based on the speed of movement should have robust outcome measures that are not influenced by the intrarater and interrater variability associated with passive movement.

We previously published a method ${ }^{22}$ for evaluating tremor during DBS surgery by using acceleration sensors. In the current study, we present various modifications of this method, with the aim of helping the neurologist assess the effect of stimulation on rigidity during intraoperative stimulation tests. Our hypothesis is that the relative change in rigidity can be assessed by measuring the acceleration of the evaluator's wrist during passive movement. To validate our approach, we studied the intra- and interrater variability of accelerometer measurements of passive movement in postoperative follow-up examinations of patients with PD treated with DBS. We evaluated the feasibility of intraoperative use of the system in a total of 9 DBS operations. The impact that this method would have had on the DBS surgery was studied by analyzing differences in the choice of position for the chronic DBS lead implantation (clinical choice vs choice based on accelerometry), a decision that is based on results of intraoperative stimulation tests. In addition, the accelerometry-based change in rigidity was correlated with the anatomical location of the stimulating electrode to identify the most effective stimulation sites.

\section{Methods \\ Recording Equipment}

A commercially available accelerometer sensor evaluation board (STEVAL-MKI022VI, STMicroelectronics) was used to measure the acceleration of the passive movements. It was housed inside a printed plastic case (FullCure 830 Vero White, Stratasys) that could be attached to the wrist with a Velcro strap (Fig. 1). The sensor board was connected through a Universal Serial Bus (USB) cable to the laptop running custom-made in-house software (LemurDBS). A wireless sensor system was not used to avoid any possible interference with the microelectrode record- 
ing (MER) of the signal. Acceleration data were recorded and visualized with LemurDBS.

\section{Filtering and Outcome Measures}

The acceleration data were processed with MATLAB version R2015b (MathWorks, Inc.). They were filtered with a smoothness priors ${ }^{24}$ filter to remove the influence of gravity. To identify the changes in rigidity during intraoperative stimulation tests, the data were analyzed in a windowed manner. Various time lengths, ranging from 1 to 4 seconds with $0 \%-50 \%$ overlap, were tested. On the basis of the duration of a single passive movement (approximately 1 second), the duration of a single stimulation current amplitude, and the sampling frequency of the accelerometer, the 4 -second window with $50 \%$ overlap was found to be optimal. To simplify the comparisons between various data sets, outcome measures were extracted from all windowed data. The measures previously found to be correlated with changes in tremor ${ }^{22}$ (standard deviation, signal energy, and spectral amplitude of the dominant frequency) were used, along with others (entropy and primary frequency). For simplicity, these outcome measures will be referred to numerically as follows: 1) standard deviation, 2) signal energy, 3) spectral amplitude of the dominant frequency, 4) dominant frequency, and 5) entropy.

\section{Data Acquisition}

\section{Reliability Tests}

To study the intra- and interrater variability of the outcome measures, it was necessary to collect data in a controlled environment. Such data collection during intraoperative stimulation tests would have prolonged the operations and put the patients under additional stress. Moreover, because of restrictions imposed by the OR environment, only 1 sensor could be used at a time to measure acceleration. Theoretically, acceleration of passive movements could be measured by attaching the sensor either to the patient or to the evaluator. We hypothesized that attaching the sensor to the evaluator would record higher-quality data than placing the sensor on the patient during passive movements of the wrist joint. To study this matter further and investigate the intra- and interrater variability of accelerometer data measured during passive movement, we performed special tests during the postoperative follow-up of patients with PD treated with DBS.

A specific protocol was set up to be followed by each evaluator when obtaining data from a patient. The evaluators were required to perform a set of 4 passive movements (left elbow, left wrist, right elbow, and right wrist) twice, with a short pause in between. Data from 2 acceleration sensors (1 attached to the patient and 1 attached to the evaluator) were recorded simultaneously and in a synchronized manner to test the quality of acceleration data obtained from the 2 fixation positions during different evaluations.

\section{Feasibility of Intraoperative Use}

To study the intraoperative feasibility of the system, it was used during DBS operations at the University Hospital in Clermont-Ferrand, France.
Surgical Procedure. The clinical team uses direct targeting on patient-specific imaging data as part of the surgical procedure. ${ }^{14}$ During the planning session, with the aid of a brain atlas developed in-house ${ }^{13}$ and multiple image sets of the patient (CT scans and MR images), including a specific white matter-attenuated inversion recovery sequence, the neurosurgeon identified and outlined the different deep brain structures in the subthalamic region with the iPlan Stereotaxy 3.0 planning software (Brainlab). Two trajectories per hemisphere were planned, from an entry point in the skull to the target structure, avoiding sensitive structures (blood vessels, cerebral ventricles). For stimulation tests, multiple positions were identified along these trajectories in the region of interest, in 1-mm steps.

The surgery was performed on awake patients. Two exploration electrodes (Alpha Omega) were inserted and electrophysiological mapping (MER) was performed at the preplanned positions in both trajectories simultaneously. After MER, stimulation tests were performed at the same positions: first in one trajectory, then in the other, moving progressively deeper into the brain tissue in steps of $1 \mathrm{~mm}$. At each position, the stimulation current was increased from 0 to $3 \mathrm{~mA}$ in steps of $0.2 \mathrm{~mA}$. To evaluate rigidity, the neurologist first passively moved the patient's arm/wrist to assess baseline rigidity, before any stimulation was delivered. Then, during stimulation, changes in rigidity compared with baseline were rated on a scale from 0 (no change) to 4 (complete suppression of rigidity). $\mathrm{Ri}-$ gidity could be rated multiple times in a single evaluation, but only the maximum reduction in rigidity was noted and used for comparison with the results at other stimulation positions. The evaluator continuously interacted with the patient to observe any side effects and to make sure that he or she was not actively moving the limb.

At each position, 2 stimulation amplitudes were identified: 1) the amplitude at which the highest reduction in rigidity was observed (subjectively assessed amplitude [AmpS]), and 2) the amplitude at which the first side effect occurred (side-effect threshold). When stimulation tests were completed, the surgical team determined the best implant position, according to the following criteria: 1) low AmpS value, 2) large difference ("therapeutic window") between AmpS and the side-effect threshold, 3) neighboring positions having a comparatively large therapeutic window, and 4) anatomical position. Depending on the number of preoperatively chosen positions, stimulation tests in 1 cerebral hemisphere can take 15-30 minutes.

Intraoperative Protocol for Data Recording. To avoid prolonging the surgical procedure while maximizing the data recorded during all of the stimulation tests, a systematic data-recording procedure was followed. The acceleration sensor was mounted on the evaluator's wrist during the stimulation tests. The strap was tightened snugly, but not uncomfortably, to prevent slippage. Data recording was started during the baseline evaluation and continued until the stimulation test for 1 position was completed. A new recording was started in a similar manner for each position, and the data were saved in separate files for offline analysis.

The stimulation current amplitude was varied in quick succession. To identify the exact amplitude responsible for 


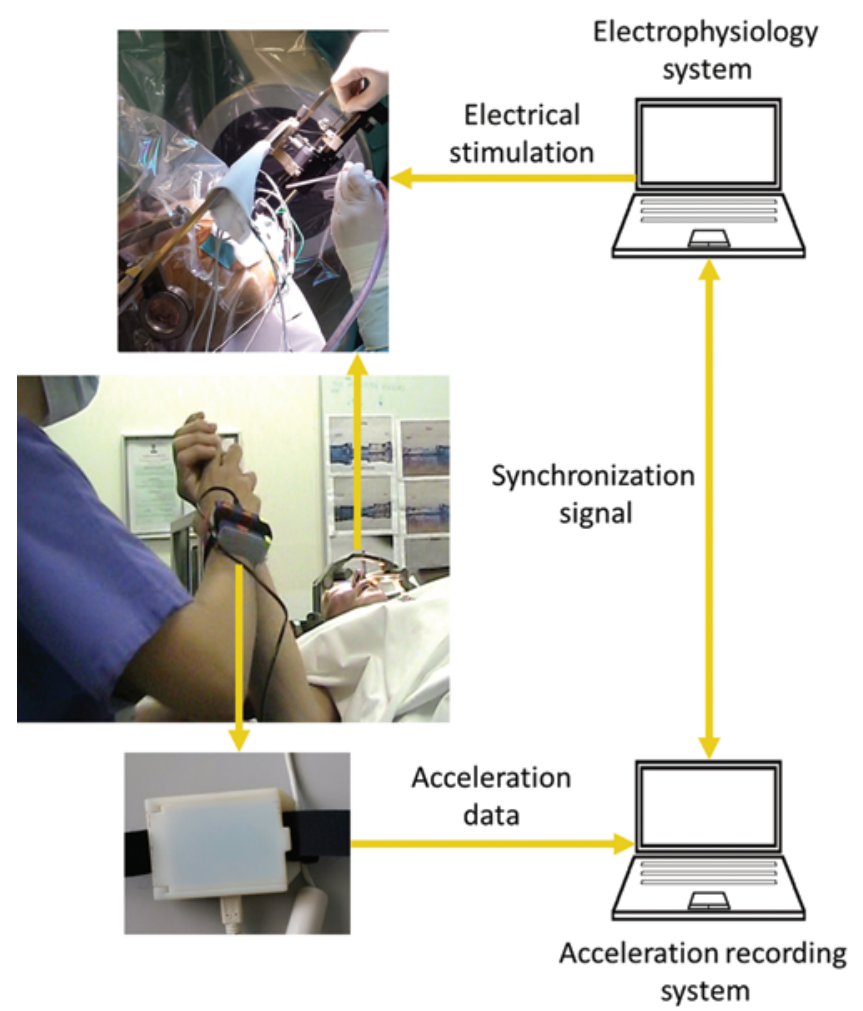

FIG. 1. Data recording setup used to assist rigidity evaluations performed during DBS surgery. Figure is available in color online only.

the therapeutic effects, recording of acceleration data was synchronized with the electrophysiological system. For this purpose, the electrophysiology system (Microguide Pro, Alpha Omega) was connected to a laptop running LemurDBS with a USB-Bayonet Neill-Concelman (USBBNC) cable (Fig. 1).

\section{Clinical Application}

The clinical use of the afore-described data-recording system for the purpose of intraoperative and postoperative symptom evaluation in the framework of this study was approved by the institutional review boards of the Univer- sity Hospital in Clermont-Ferrand (France) and the University Hospital of Basel (Switzerland).

For the tests of intra- and interrater reliability in postoperative follow-up, 3 different evaluators were asked to perform passive movements to assess rigidity in 3 patients with PD, according to a predefined protocol. To simulate OR conditions, the tests were done with the patients supine. Each evaluator completed 8 passive movements for each patient, yielding a total of 72 evaluations ( 3 patients $\times$ 3 evaluators $\times 2$ sides $\times 2$ joints $\times 2$ evaluations).

To investigate the feasibility of using the system during intraoperative stimulation tests, the recording system was used during the DBS operations of 9 patients with PD, who had rigidity as their primary symptom. The patients gave their written informed consent to participate in the study (for details, see Table 1). Four different neurologists performed routine passive movements to evaluate rigidity while wearing the acceleration sensor on their wrist (each patient was evaluated intraoperatively by only 1 neurologist).

\section{Data Analysis Method}

The data were analyzed with MATLAB. Statistics were performed with SOFA Statistics version 1.4.5 (Paton-Simpson \& Associates Ltd.) and OriginPro (OriginLab Corporation).

\section{Reliability Tests}

To validate the outcome measures, they were extracted from the acceleration sensors in both positions (attached to the patient and to the evaluator, respectively) with the MATLAB scripts after verification of synchronization. Because these measures were extracted in a windowed fashion, multiple sets of measures were available per evaluation. The outcome measures extracted from the sensors on the patient and on the evaluator would be expected to be strongly correlated with each other (pairwise) because of the simultaneous synchronized recording of data. An absence of correlation would indicate insufficient quality of acceleration data. For each evaluation, the multiple sets of outcome measures were averaged to create a pairwise data set. To confirm our presumption that the sensor on

TABLE 1. Details about patients participating in the clinical study and their surgical procedures

\begin{tabular}{cccclc}
\hline \multirow{2}{*}{$\begin{array}{c}\text { Patient } \\
\text { No. }\end{array}$} & Age at & Preop UPDRS-III & Evaluator & \multicolumn{2}{c}{ Tested Trajectories (no. of stimulation positions) } \\
\cline { 6 - 6 } & Surgery (yrs) & Scores (medication off) & ID & & Lt \\
\hline 1 & 67 & 17 & E1 & Central (9), posterolateral (9) & Central (8), posterolateral (8) \\
\hline 2 & 60 & 26 & E1 & Central (8), posterior (8) & Central (3), posterior (3) \\
\hline 3 & 53 & 39.5 & E1 & Central (4), posterior (4) & Central (8), posterior (8) \\
\hline 4 & 61 & 26 & E4 & Central (8), posterior (8) & Central (6), posterior (6) \\
\hline 5 & 66 & 35 & E3 & Central (8), posterior (8) & Central (7), posterior (7) \\
\hline 6 & 69 & 45 & E2 & Central (6), posterior (6) & Central (6), posterior (6) \\
\hline 7 & 57 & 45 & E4 & Central (8), posterior (8) & Central (8), posterior (8) \\
\hline 8 & 69 & 27 & E2 & Central (7), posterior (7) & Central (7), posterior (7) \\
\hline 9 & 53 & 43 & E2 & Central (7), posterolateral (7) & Central (7), posterolateral (7) \\
\hline
\end{tabular}

UPDRS = Unified Parkinson's Disease Rating Scale

Acceleration data were unavailable for the stimulation tests in boldface type. 
TABLE 2. Categorization, comparison, and statistical tests used for analysis of data collected during reliability tests

\begin{tabular}{ccccc}
\hline Purpose & Data Constraints \& Groups & Comparison & Test & Result \\
\hline $\begin{array}{c}\text { Patient sensor } \\
\text { vs evaluator } \\
\text { sensor }\end{array}$ & $\begin{array}{c}\text { Test 1: elbow movements; } \\
\text { Test 2: wrist movements }\end{array}$ & $\begin{array}{c}\text { Pairwise correlation of outcome } \\
\text { measures from patient's sen- } \\
\text { sor \& evaluator's sensor }\end{array}$ & $\begin{array}{c}\text { Spearman's cor- } \\
\text { relation test }\end{array}$ & $\begin{array}{c}\text { Correlation for elbow movements (Test 1): } \\
\text { outcome measures 1, 2, \& 3; correlation } \\
\text { for wrist movements (Test 2): none }\end{array}$ \\
\hline $\begin{array}{c}\text { Intrarater vari- } \\
\text { ability }\end{array}$ & $\begin{array}{c}\text { Data only from evaluator's } \\
\text { sensor }\end{array}$ & $\begin{array}{c}\text { Pairwise comparison btwn out- } \\
\text { come measures of repeated } \\
\text { evaluation of the same joint }\end{array}$ & $\begin{array}{c}\text { Paired-sample Wil- } \\
\text { coxon signed- } \\
\text { rank test }\end{array}$ & No change in outcome measures 1, 2, \& 3 \\
\hline $\begin{array}{c}\text { Interrater vari- } \\
\text { ability }\end{array}$ & $\begin{array}{c}\text { Data only from evaluator's } \\
\text { sensor. Comparison per } \\
\text { patient, side, \& joint }\end{array}$ & $\begin{array}{c}\text { Comparison of outcome mea- } \\
\text { sures 1, 2, \& 3 btwn different } \\
\text { evaluators }\end{array}$ & $\begin{array}{c}\text { Kruskal-Wallis } \\
\text { ANOVA }\end{array}$ & $\begin{array}{c}\text { Interrater variability in outcome measures } \\
1,2, \& 3\end{array}$ \\
\hline
\end{tabular}

the patient would not be sensitive enough to detect wrist movements, the pairwise correlation of the outcome measures from the patient's and the evaluator's sensors was tested for wrist and elbow evaluations separately with the Spearman correlation test.

For reliable assessment of changes in rigidity, the outcome measures of the evaluator's sensor were checked for intrarater variability by comparing data from evaluations 1 and 2 of each evaluation type. From the data set of averaged outcome measures, only those for the evaluator's sensor were separated into data from evaluations 1 and 2 for pairwise comparison. The Wilcoxon signed-rank test was used to verify similarity between the outcome measures of these 2 groups.

To study interrater variability, the multiple outcome measures per evaluation were compared independently for the different evaluators and patients. The data were grouped for the 4 different joints (left elbow, left wrist, right elbow, and right wrist). The Kruskal-Wallis ANOVA test was used for each patient to check for interrater variations.

The different groupings and statistical tests described above are summarized in Table 2 .

\section{Feasibility of Intraoperative Use}

The intraoperatively recorded acceleration data were synchronized with the stimulation current amplitude according to the time-stamped synchronization signal (Fig. 2 , upper). The repetitive nature of passive movements facilitated the identification and removal of data corresponding to other movements of the evaluator's arm. The data corresponding to 1 stimulation test were divided into 2 parts as follows: 1) the data recorded during passive movements without any stimulation were called baseline data, and 2) the remaining data were called stimulation data. These data were then filtered and outcome measures were extracted with the MATLAB scripts as described above. Further, to perform comparative analysis in a manner similar to the neurologists' evaluations, the extracted outcome measures from the stimulation data were normalized to the highest outcome measures at baseline (Fig. 2, lower).

To investigate the pairwise correlation between normalized outcome measures and clinical ratings, Spearman's correlation test was used. Because a neurologist's assessment of change in rigidity was only noted once for each stimulation position (change at AmpS), the subjective ratings were compared with the normalized outcome mea- sures at AmpS. Furthermore, the mean of the normalized outcome measures that were well correlated was called the quantitatively assessed change (QC) in rigidity.

\section{Identification of Rigidity Release Threshold}

During subjective evaluations, the AmpS (that is, the stimulation current amplitude for the highest change in rigidity) was determined by the evaluator for each stimulation position. In addition, the amplitude inducing a QC similar to the subjectively assessed reduction at AmpS was identified and designated as quantitatively identified rigidity-suppressing amplitude (AmpQ). The Wilcoxon 2-sided signed-rank test was used to compare AmpS and
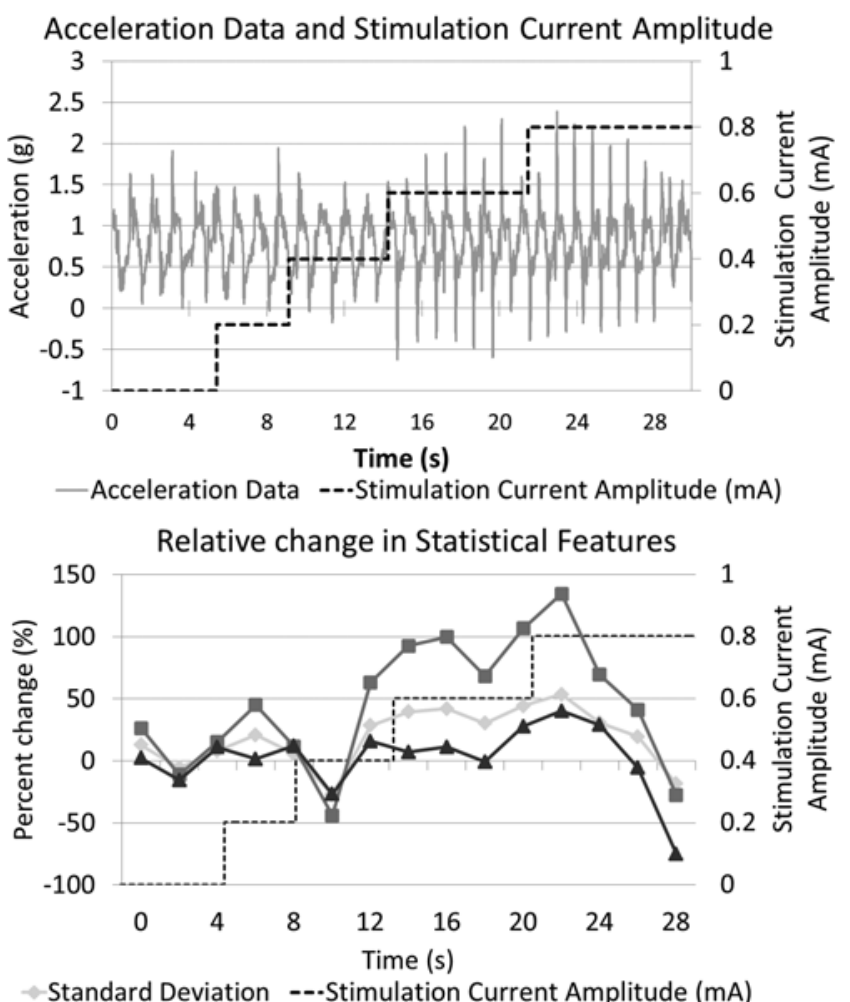

-Signal Energy $\quad$-Spectral amplitude of primary frequency

FIG. 2. Graphical representation of the recorded acceleration data synchronized with the stimulation current amplitude (upper) for 1 stimulation test, and outcome measures extracted in a windowed manner from the recorded signal along with the stimulation current amplitude (lower). 
AmpQ for stimulation tests where both the AmpQ and the AmpS were identified.

\section{Impact on Surgical Decision Making}

During the routine procedure, the surgical team would determine the best implant position of the DBS lead on the basis of the AmpS, the side-effect threshold, and the anatomy, as described above. To study the effect of accelerometry-based rigidity change assessment on the final implant position chosen, the clinical staff was asked which final implant position they would have chosen if they had considered the AmpQ rather than the AmpS values. These choices were compared with the actual best implant positions chosen after intraoperative stimulation testing for each patient.

\section{Anatomical Analysis}

The intraoperative accelerometric assessment of change in rigidity was used to study the efficacy of stimulation in different anatomical structures as targets for the reduction of rigidity. On the basis of the planning data, the surgical team postoperatively identified the anatomical structure present at the center of the stimulating contact for every stimulation position and verified it with the MER data. To compare the efficacy of the different anatomical locations on the basis of the accelerometric measurements, at each stimulation test position the lowest stimulation current amplitude at which the QC value increased to more than $75 \%$ was identified, if it existed (Amp75). The threshold value of $75 \%$ was chosen to identify only the structures responsible for high reduction of rigidity. We also compared the side-effect thresholds and the number of stimulation tests where side effects were observed as a percentage of the total number of stimulation tests in a particular structure. These data sets, composed of Amp75, occurrence of side effects, and side-effect thresholds, were used to compare the efficacies of different anatomical structures.

\section{Results}

The accelerometric method could be successfully applied in the postoperative validation tests and in 9 DBS operations without prolonging the procedures. All evaluators and patients were comfortable with wearing the acceleration sensor while performing the rigidity evaluations. Routine evaluations and routine surgical procedures took place as usual, without any interruptions. During implantation in the left hemisphere of Patient 1, the sensor was mounted on the patient's wrist and not on the evaluator's. Data recorded during these stimulation tests were not included in the analysis. No electrophysiological data were available for implantation in the left hemisphere of Patient 3. For Patient 7, due to the absence of rigidity, akinesia was evaluated during stimulation tests in the left hemisphere.

\section{Reliability Tests}

Figure 3 shows an example of a comparison between data sets acquired with acceleration sensors mounted on the patient and on the evaluator for elbow and wrist movements. For the elbow tests, of the 5 outcome measures, 3 showed a strong correlation (outcome measure $1: \mathrm{R}=$ $0.766, \mathrm{p}<0.001$; outcome measure $2: \mathrm{R}=0.756, \mathrm{p}<0.001$;
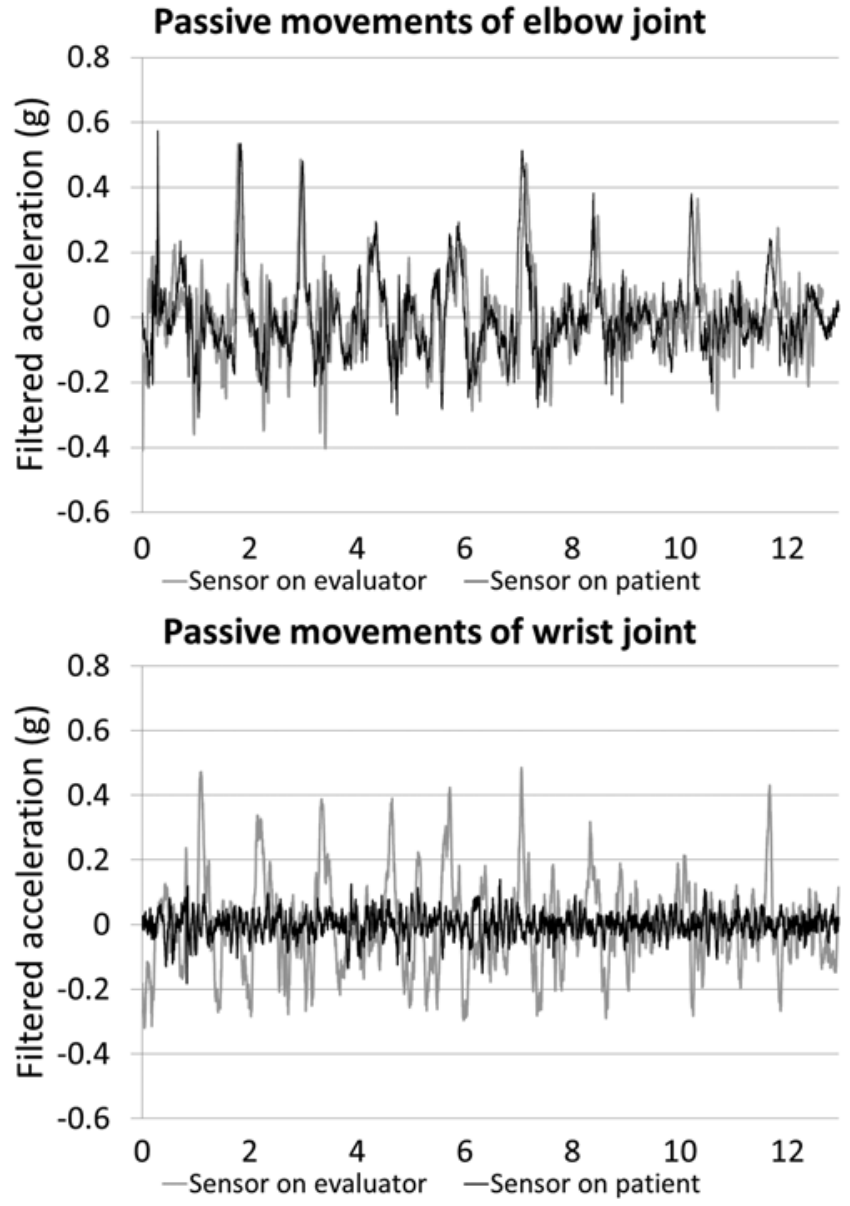

FIG. 3. Filtered acceleration data from the sensors mounted on the evaluator (solid gray line) and on the patient (solid black line). The graphs show data from the passive movements of the elbow (upper) and wrist joint (lower).

and outcome measure 3: $\mathrm{R}=0.697, \mathrm{p}<0.001$ ) between the 2 sensors, whereas the remaining outcome measures (4 and 5) showed a weak or no correlation. In contrast, none of the features exhibited a correlation between patientderived and evaluator-derived measurements of passive movement of the wrist.

In the analysis of intrarater variability, the results of the paired-sample Wilcoxon signed-rank tests showed that 3 outcome measures did not change (outcome measures 1, 2, and $3 ; \mathrm{p}<0.001$ ) between repeated evaluations of the same joint. Therefore, only these 3 outcome measures were retained in the further analysis.

The interrater variability analysis (Kruskal-Wallis ANOVA test) for the different joints and for each patient showed that outcome measures 1,2 , and 3 were significantly different $(\mathrm{p}<0.05)$ among the 3 evaluators. Box plots in Fig. 4 represent the interrater variations in outcome measure 1. They show that for some evaluations (left elbow, right elbow, and right wrist of Patient 3), the interrater variability was very low, but for others (left and right elbow of Patient 1, and right wrist of Patient 3) there were significant interrater differences. Outcome measures 2 and 3 have similar distributions. 

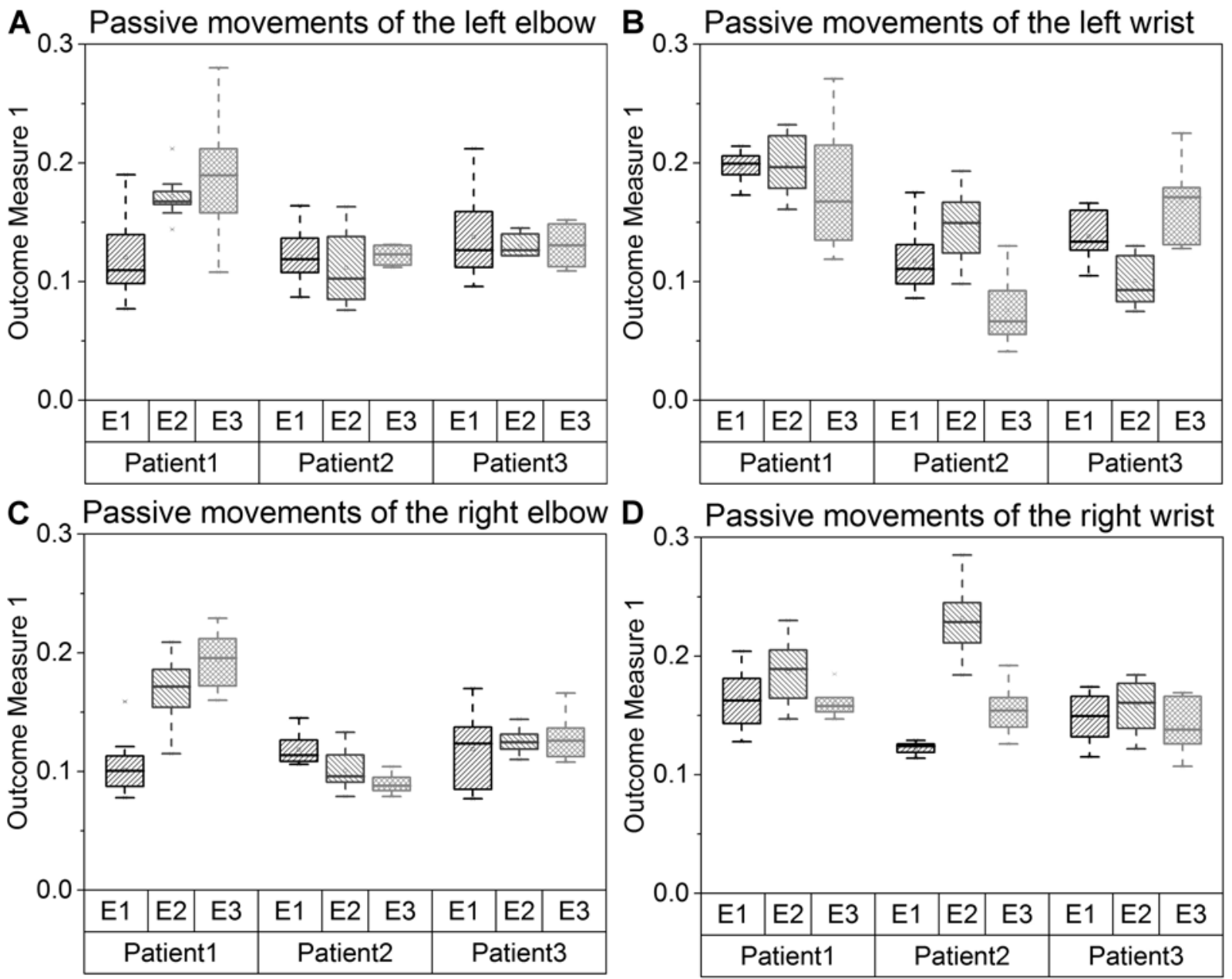

FIG. 4. Box plots showing the interrater variations (E1, E2, E3) for outcome measure 1 for passive movements of the left elbow (A), left wrist (B), right elbow (C), and right wrist (D).

The statistical results of the above tests are summarized in Table 2.

\section{Feasibility of Intraoperative Use}

The comparison between the normalized outcome measures and the subjectively rated change in rigidity during intraoperative stimulation tests indicates a strong correlation for outcome measures $1(\mathrm{R}=0.308, \mathrm{p}<0.001), 2$ ( $\mathrm{R}$ $=0.313, p<0.001)$, and $3(\mathrm{R}=0.237, \mathrm{p}<0.05)$. Therefore, QC (the quantitatively assessed change in rigidity) was calculated as the mean of the normalized values of these outcome measures.

\section{Identification of Rigidity Release Threshold}

Of 188 stimulation tests during which acceleration data were recorded, AmpS and AmpQ were available and could be compared for 140 stimulation tests. The other 48 were composed of 14 evaluations where neither method identified any reduction in rigidity, 30 evaluations where the subjective evaluation did not find any threshold, and 4 evaluations where the accelerometric evaluation did not identify a threshold. The mean value of AmpQ $(0.9 \pm 0.6 \mathrm{~mA})$ was lower than AmpS $(1.4 \pm 0.6 \mathrm{~mA})$, and the results of the
Wilcoxon 2-sided signed-rank test confirmed inequality between AmpQ and AmpS values (Fig. 5, p < 0.001). For stimulation tests where the value for AmpQ was lower than for AmpS, it was observed that the value of QC during stimulation was uniform between these 2 amplitudes.

\section{Impact on Surgical Decision Making}

The comparison of choices of the final implant position is shown in Table 3. Because of error in the synchronization setup, only 2 stimulation tests were analyzed for the left implantation in Patient 5, for which data were insufficient to identify the best implant position for this hemisphere. Of the remaining 14 implantations, for only 2 positions (rows in boldface type in Table 3), the choice was the same when made with AmpS or AmpQ. In 4 cases, a position on another trajectory was chosen on the basis of the AmpQ compared with the AmpS values.

\section{Anatomical Analysis}

For this analysis, 125 stimulation tests were used for which QC values were higher than $75 \%$. Furthermore, the number of stimulation tests in the substantia nigra (1 test) and thalamus (4 tests) were not large enough to provide 


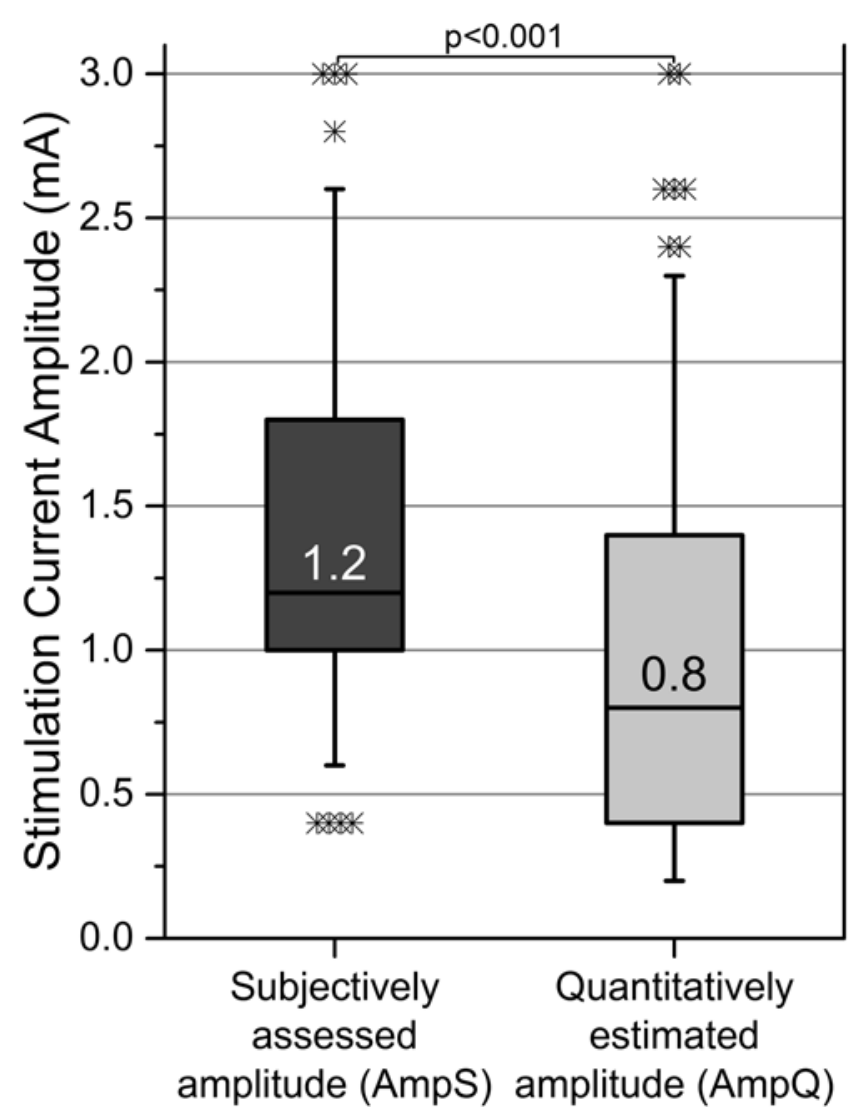

FIG. 5. Box plot comparing the rigidity-suppressing stimulation current amplitudes $(n=140)$ identified with subjective evaluation (AmpS) and from the quantitative data (AmpQ). Upper whiskers are at the 95th percentile and lower whiskers are at the 5th percentile. Asterisks represent outliers.

statistically significant results. Of the remaining 120 stimulation tests, 64 were in the STN, 35 were in the zona incerta (ZI), and 21 were found in the fields of Forel (FF). The results depicted on the left in Fig. 6 show that the STN requires lower stimulation current amplitudes than the $\mathrm{ZI}$ and FF to reduce rigidity by at least $75 \%$. The average values of side-effect thresholds were similar for all 3 structures (STN $2.68 \pm 0.36 \mathrm{~mA}, \mathrm{ZI} 2.72 \pm 0.17 \mathrm{~mA}$, and FF $2.5 \pm 0.22 \mathrm{~mA}$ ), but the STN had a significantly higher occurrence of side effects (Fig. 6, right).

\section{Discussion}

This article describes a novel method of recording and analyzing rigidity evaluation during DBS surgery by means of an acceleration sensor strapped on the evaluator's wrist. The aim was to test the hypothesis that changes in the patient's rigidity can be assessed by measuring acceleration during passive movement. Validation tests were performed to confirm the optimal mounting position of the acceleration sensor, to check for low intrarater variability of the outcome measures, and to assess interrater variability, before using the system intraoperatively.

\section{Reliability Tests}

The comparison between the data of the sensor on the patient's wrist and the sensor on the evaluator's wrist (Fig. 3 ) and the Spearman's correlation test confirmed our hypothesis that a fixation on the evaluator's wrist is more robust for measuring passive movements of both joints. Therefore, for an intraoperative use without altering the existing surgical procedure, the sensor should be mounted on the wrist of the evaluator, as done in the current study.

The low intrarater variability in 3 of the 5 outcome measures shows that these outcome measures are sufficiently robust for use in the intraoperative environment. During our intraoperative stimulation tests, intrarater variability would not have been relevant because the evaluator performs no other activity than passive movement, pausing only between stimulation test positions. In any case, even if the evaluator has to stop and then resume passive movements, the current method will be able to reliably assess the change in rigidity.

As mentioned previously, the performed passive movements vary between evaluators, ${ }^{27}$ so interrater variations are expected in the accelerometer data. These variations also influence the outcome measures, as depicted in the box plots in Fig. 4 and as statistically confirmed. It must be borne in mind that the results of the Kruskal-Wallis ANOVA test are based on a small sample size, thus reducing their power. Because of the high inherent interrater variability of passive movements, the determination of the absolute level of rigidity in patients by measuring the acceleration is not possible. Thus, the aim of the current study was to develop a method for the determination of relative changes compared with baseline evaluations.

\section{Feasibility of Intraoperative Use}

The method proposed in this article is designed to assist the neurologist in estimating the changes in rigidity during intraoperative stimulation tests of DBS surgery. Considering that in most centers only 1 evaluator is present to perform the passive movements, the current method will produce reliable results because of the high intrarater reliability. Additionally, the technique of normalization of the outcome measures used in the current method not only mimics the subjective evaluation method (that is, assess changes compared with baseline values), but also minimizes the effect of any residual intrarater variability between different stimulation test positions. The evidence is provided by the results of the Spearman's correlation tests between the 3 normalized outcome measures and the clinically rated changes in rigidity.

Moreover, such an evaluation is superior to absolute evaluation, because the rigidity of the patient may also change due to other factors such as microlesional effects, patient stress, and so on. However, it was also noted during the data analysis that a minimum recording of 5 seconds of baseline evaluation is needed to obtain evaluable results. When such data were unavailable, the baseline data from the previous stimulation test position (a maximum of 3 minutes before) were used. The results of the application of this method in 9 DBS operations support its future use in the OR.

Quantitative rigidity evaluations have rarely been performed during DBS surgery. To our knowledge, only Kwon et al. ${ }^{2}$ have used a mechanical setup to measure parameters of rigidity in the wrist joint while the patient 
TABLE 3. Choice of best implant position for the DBS lead

\begin{tabular}{|c|c|c|c|c|c|}
\hline \multirow{2}{*}{$\begin{array}{c}\text { Patient } \\
\text { No. }\end{array}$} & \multirow[b]{2}{*}{ Hemisphere } & \multicolumn{2}{|c|}{ Choice Based on Clinical Evaluation } & \multicolumn{2}{|c|}{ Choice Based on Thresholds Extracted From Acceleration Data } \\
\hline & & Trajectory & Distance to Target (mm) & Trajectory & Distance to Target $(\mathrm{mm})$ \\
\hline 1 & Lt & Central & 0 & NA & NA \\
\hline 1 & Rt & Central & -1 & Central & -3 \\
\hline 2 & Lt & Central & 0 & Posterior & -1 \\
\hline 2 & Rt & Central & -3.5 & Posterior & -4 \\
\hline 3 & $\mathrm{Lt}$ & Central & -4 & NA & NA \\
\hline 3 & Rt & Central & -3 & Central & -3 \\
\hline 4 & Lt & Central & -1 & Central & -3 \\
\hline 4 & $\mathrm{Rt}$ & Central & -2 & Central & -3 \\
\hline 5 & Lt & Central & -4.5 & NA & NA \\
\hline 5 & Rt & Central & -1 & Central & -1 \\
\hline 6 & $\mathrm{Lt}$ & Central & -3.5 & Central & -3 \\
\hline 6 & $\mathrm{Rt}$ & Central & -3.5 & Posterolateral & -3 \\
\hline 7 & $\mathrm{Rt}$ & Central & -2 & Posterolateral & -5 \\
\hline 8 & $\mathrm{Lt}$ & Central & -2 & Central & -1 \\
\hline 8 & Rt & Central & -2 & Central & -6 \\
\hline 9 & $\mathrm{Lt}$ & Central & -2.5 & Central & -1 \\
\hline 9 & Rt & Central & -3.5 & Central & -8 \\
\hline
\end{tabular}

NA = not available (that is, for stimulation tests in the left hemisphere of Patients 1,3 , and 5, acceleration data were insufficient to make a choice).

The choice of the best implant position was the same for the rows in boldface type.

underwent surgery. An expert evaluator passively moved the patient's wrist around the joint through a handle while the input force of the evaluator and the inertia of the movement were measured. Mechanical parameters such as work, impulse impedance, and so on were extracted. In contrast to our method, their setup enables the measurement of multiple variables simultaneously, but is restricted to evaluation of the wrist joint. Moreover, for proper functioning, instructions were given to the evaluator to perform a specific movement, which resulted in longer evaluations. In addition, the evaluator had to change his or her routine evaluation protocol. In order not to prolong the surgical procedure, fewer evaluations were performed when their setup was used. ${ }^{12}$ Our system was specifically designed for use in routine evaluation; no instructions were given to the patient or the evaluator. Because the evaluator could follow his or her usual evaluation protocol, the duration of the routine surgery was unaltered.

\section{Identification of Rigidity Release Threshold}

One of the advantages of using our method rather than subjective evaluation is in the identification of rigidity release thresholds. Twenty-six more thresholds were found with our data analysis technique. Also, the results of the

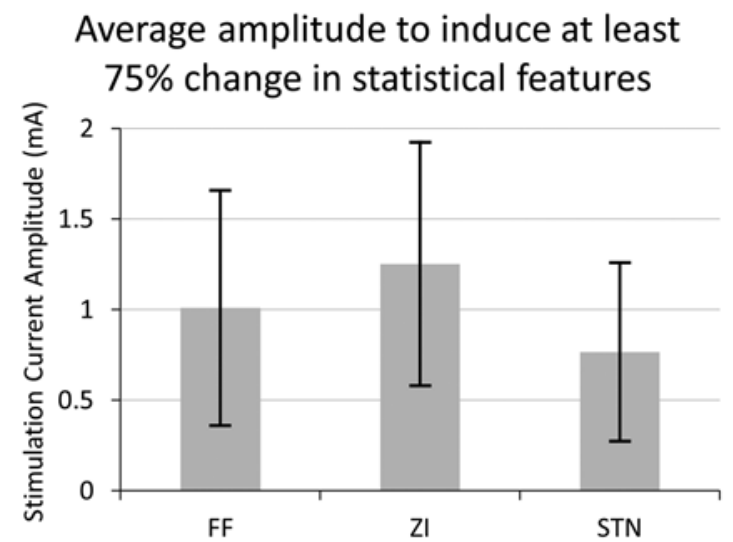

\section{Stimulation tests with and without side-effect occurrences}

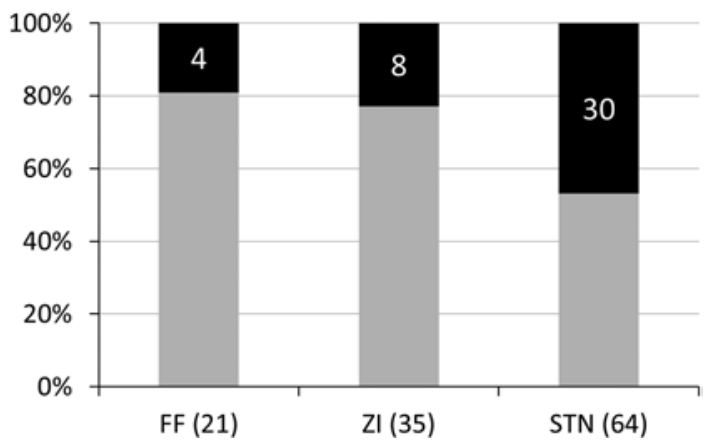

FIG. 6. Left: Column charts comparing the average stimulation current amplitude required to observe at least a $75 \%$ reduction in the mean of the 3 outcome measures (standard deviation, signal energy, and spectral amplitude of the primary frequency). Right: Stacked $100 \%$ column charts present the number of stimulation tests where side effects were observed (black) as a percentage of the total number of stimulation tests in the corresponding structure. The numbers inside the black part of the bars indicate the actual count of the side-effect occurrences; the numbers below the bars indicate the total number of stimulation tests. 
Wilcoxon 2-sided signed-rank test and the box plot show that values tend to be lower for AmpQ than for AmpS. We believe that the human evaluator, on observing the change in rigidity, may need a few seconds to verify and assess the amount of reduction. During this time, the amplitude may have already been increased, resulting in higher AmpS values. This result suggests that the current setup enhances the sensitivity of the subjective rigidity evaluation. On the other hand, there were 4 positions where AmpS values, but no AmpQ values, were found. On inspection of these 4 positions, it was observed that the baseline data were insufficient to extract outcome measures for normalization. Because they were the first positions tested, baseline evaluations of previous positions were unavailable to use the workaround. This underscores the need for sufficient baseline recording for data analysis.

\section{Impact on Surgical Decision Making}

The differences in the rigidity release amplitudes identified by the 2 methods significantly influenced the choice of the best implant position. Only 2 of the 12 choices would have been at the same position. This clearly shows the potential of using an assistive method during intraoperative symptom evaluation for DBS surgery. From the current data, it is not possible to judge which of the 2 choices was better. One possible way would be to compare the choice of best implant position with the choice of postoperative contact; the relative closeness of one or the other choice may help to judge whether the assistive evaluation system can identify better implant positions. However, such an analysis would need a very large data set to result in statistically significant conclusions.

\section{Anatomical Analysis}

The anatomical analysis gives interesting insight into the target selection procedure of DBS. It shows that stimulation in the STN requires the lowest current amplitude to achieve a $75 \%$ reduction in rigidity. However, in view of the fact that side effects were observed in nearly $50 \%$ of stimulations in the STN, it may not be the optimum target structure. The FF require an additional $0.3 \mathrm{~mA}$, on average, to achieve a comparable reduction in rigidity, but have significantly fewer side-effect occurrences. Hence, to achieve a better outcome of DBS for patients with rigidity, the DBS lead might be placed closer to the FF or the ZI and not inside the STN, to avoid side effects. This would be in accordance with reported findings $\mathrm{s}^{3,6,9,20,28}$ implying that the FF and the ZI may also be responsible for the therapeutic effects of DBS. However, this analysis has a limitation. It only considers the center of the electrode when associating a structure with a stimulation test position. Because the effect of stimulation spreads beyond the center, electric-field simulations would be a helpful tool to deepen the analysis of the available intraoperative data.

\section{Future Work}

In its current state, the method proposed in this study has various advantages over the existing subjective ratings used in the OR. It provides a reproducible assessment of the change in rigidity, which can be reviewed at any time dur- ing surgery without any discomfort to the patient or evaluator, and without prolonging the operation. Nevertheless, because the changes in rigidity are assessed indirectly, the results produced are only semiquantitative. In the future, to make the method a comprehensive evaluation system for use in DBS surgery, the current setup will be modified to enable data recording and analysis from 2 smaller acceleration sensors simultaneously. By mounting one sensor on the evaluator and the other on the patient, ${ }^{23}$ it will be possible to evaluate rigidity and tremor at the same time. We intend to use the system in more operations (optimally, in additional clinical centers as well) so that we can continue to adapt it to physicians' needs. The long-term aim is to integrate this method in commercially available and currently used intraoperative stimulation systems to facilitate visualization of the symptom improvement in real time, along with other stimulation parameters and anatomical data. Such a system would enable the surgical team to interpret the results of intraoperative stimulation tests more easily and thus facilitate surgical decision making.

\section{Conclusions}

We have described a new way to assess changes in rigidity by measuring the acceleration of the examiner's wrist during passive movement while the patient is undergoing intraoperative stimulation testing in DBS surgery. Outcome measures are reproducible over time but are influenced by variations in the passive movements of different evaluators. The intraoperatively assessed change in rigidity is reliable and is correlated with the subjective ratings. The quantitatively identified effective stimulation amplitudes show the potential of the method to optimize surgical decision making. Moreover, our findings concerning the thresholds for effective stimulation at different anatomical sites suggest that the optimal DBS target may, in fact, lie closer to the FF, just dorsal to the STN. The proposed method may be a clinically and scientifically useful aid to optimal targeting in DBS.

\section{Acknowledgments}

This research was funded by the Swiss National Science Foundation as a part of the project 205321_135285 and partly by the Germaine de Stael program of the Swiss Academy of Engineering Sciences. We acknowledge and thank Prof. F. Durif, Dr. M. Ulla, and Dr. A. Marques of the Department of Neurology at the CHU Clermont-Ferrand, France, and Ms. Bianca Raffaelli and Prof. Dr. Peter Fuhr of the Department of Neurology at the University Hospital of Basel, Switzerland, for performing the routine passive movements for rigidity evaluations.

\section{References}

1. Abosch A, Timmermann L, Bartley S, Rietkerk HG, Whiting D, Connolly PJ, et al: An international survey of deep brain stimulation procedural steps. Stereotact Funct Neurosurg 91:1-11, 2013

2. Askari S, Zhang M, Won DS: An EMG-based system for continuous monitoring of clinical efficacy of Parkinson's disease treatments. Conf Proc IEEE Eng Med Biol Soc 2010:98-101, 2010

3. Aström M, Tripoliti E, Hariz MI, Zrinzo LU, Martinez-Torres I, Limousin P, et al: Patient-specific model-based investiga- 
tion of speech intelligibility and movement during deep brain stimulation. Stereotact Funct Neurosurg 88:224-233, 2010

4. Endo T, Okuno R, Yokoe M, Akazawa K, Sakoda S: A novel method for systematic analysis of rigidity in Parkinson's disease. Mov Disord 24:2218-2224, 2009

5. Fung VS, Burne JA, Morris JG: Objective quantification of resting and activated parkinsonian rigidity: a comparison of angular impulse and work scores. Mov Disord 15:48-55, 2000

6. Godinho F, Thobois S, Magnin M, Guenot M, Polo G, Benatru I, et al: Subthalamic nucleus stimulation in Parkinson's disease: anatomical and electrophysiological localization of active contacts. J Neurol 253:1347-1355, 2006

7. Goetz CG, Tilley BC, Shaftman SR, Stebbins GT, Fahn S, Martinez-Martin P, et al: Movement Disorder Societysponsored revision of the Unified Parkinson's Disease Rating Scale (MDS-UPDRS): scale presentation and clinimetric testing results. Mov Disord 23:2129-2170, 2008

8. Griffiths RI, Kotschet K, Arfon S, Xu ZM, Johnson W, Drago $\mathrm{J}$, et al: Automated assessment of bradykinesia and dyskinesia in Parkinson's disease. J Parkinsons Dis 2:47-55, 2012

9. Hamel W, Fietzek U, Morsnowski A, Schrader B, Herzog J, Weinert D, et al: Deep brain stimulation of the subthalamic nucleus in Parkinson's disease: evaluation of active electrode contacts. J Neurol Neurosurg Psychiatry 74:1036-1046, 2003

10. Hoehn MM, Yahr MD: Parkinsonism: onset, progression and mortality. Neurology 17:427-442, 1967

11. Kirollos C, Charlett A, O’Neill CJ, Kosik R, Mozol K, Purkiss AG, et al: Objective measurement of activation of rigidity: diagnostic, pathogenetic and therapeutic implications in parkinsonism. Br J Clin Pharmacol 41:557-564, 1996

12. Kwon Y, Park SH, Kim JW, Ho Y, Jeon HM, Bang MJ, et al: Quantitative evaluation of parkinsonian rigidity during intra-operative deep brain stimulation. Biomed Mater Eng 24:2273-2281, 2014

13. Lemaire JJ, Caire F, Bony JM, Kemeny JL, Villeger A, Chazal J: Contribution of 4.7 Tesla MRI in the analysis of the MRI anatomy of the human subthalamic area. Acta Neurochir (Wien) 146:906-907, 2004

14. Lemaire JJ, Coste J, Ouchchane L, Hemm S, Derost P, Ulla $\mathrm{M}$, et al: MRI anatomical mapping and direct stereotactic targeting in the subthalamic region: functional and anatomical correspondence in Parkinson's disease. Int J Comput Assist Radiol Surg 2:75-85, 2007

15. Levin J, Krafczyk S, Valkovic P, Eggert T, Claassen J, Bötzel $\mathrm{K}$ : Objective measurement of muscle rigidity in Parkinsonian patients treated with subthalamic stimulation. Mov Disord 24:57-63, 2009

16. Palmer JL, Coats MA, Roe CM, Hanko SM, Xiong C, Morris JC: Unified Parkinson's Disease Rating Scale-Motor Exam: inter-rater reliability of advanced practice nurse and neurologist assessments. J Adv Nurs 66:1382-1387, 2010

17. Park BK, Kwon Y, Kim JW, Lee JH, Eom GM, Koh SB, et al: Analysis of viscoelastic properties of wrist joint for quantification of parkinsonian rigidity. IEEE Trans Neural Syst Rehabil Eng 19:167-176, 2011

18. Post B, Merkus MP, de Bie RM, de Haan RJ, Speelman JD: Unified Parkinson's disease rating scale motor examination: are ratings of nurses, residents in neurology, and movement disorders specialists interchangeable? Mov Disord 20:15771584,2005

19. Prochazka A, Bennett DJ, Stephens MJ, Patrick SK, SearsDuru R, Roberts T, et al: Measurement of rigidity in Parkinson's disease. Mov Disord 12:24-32, 1997

20. Saint-Cyr JA, Hoque T, Pereira LCM, Dostrovsky JO, Hutchison WD, Mikulis DJ, et al: Localization of clinically effective stimulating electrodes in the human subthalamic nucleus on magnetic resonance imaging. J Neurosurg 97:1152-1166, 2002
21. Sepehri B, Esteki A, Ebrahimi-Takamjani E, Shahidi GA Khamseh F, Moinodin M: Quantification of rigidity in Parkinson's disease. Ann Biomed Eng 35:2196-2203, 2007

22. Shah A, Coste J, Lemaire JJ, Schkommodau E, Hemm-Ode $\mathrm{S}$ : A method to quantitatively evaluate changes in tremor during deep brain stimulation surgery, in 6th Annual International IEEE EMBS Conference on Neural Engineering, San Diego, 2013 (http://ieeexplore.ieee.org/ abstract/document/6696155/) [Accessed November 30, 2016]

23. Strauss I, Kalia SK, Lozano AM: Where are we with surgical therapies for Parkinson's disease? Parkinsonism Relat Disord 20 (Suppl 1):S187-S191, 2014

24. Tarvainen MP, Ranta-Aho PO, Karjalainen PA: An advanced detrending method with application to HRV analysis. IEEE Trans Biomed Eng 49:172-175, 2002

25. Wichmann T, Delong MR: Deep-brain stimulation for basal ganglia disorders. Basal Ganglia 1:65-77, 2011

26. Wright D, Nakamura K, Maeda T, Kutsuzawa K, Miyawaki K, Nagata K: Research and development of a portable device to quantify muscle tone in patients with Parkinsons disease. Conf Proc IEEE Eng Med Biol Soc 2008:2825-2827, 2008

27. Xia R: Physiological and biomechanical analyses of rigidity in Parkinson's disease, in Rana AQ (ed): Etiology and Pathophysiology of Parkinson's Disease. Rijeka, Croatia: InTech, 2011, pp 485-506

28. Zheng Z, Zhang YQ, Li JY, Zhang XH, Zhuang P, Li YJ: Subthalamic deep brain stimulation for Parkinson's disease: correlation of active contacts and electrophysiologically mapped subthalamic nucleus. Chin Med J (Engl) 122:24192422,2009

\section{Disclosures}

The authors report no conflict of interest concerning the materials or methods used in this study or the findings specified in this paper.

\section{Author Contributions}

Conception and design: Hemm, Lemaire, Schkommodau. Acquisition of data: Shah, Coste, Lemaire, Derost, Taub. Analysis and interpretation of data: Hemm, Shah, Coste, Schkommodau, Taub, Guzman. Drafting the article: Shah. Critically revising the article: Hemm, Shah. Reviewed submitted version of manuscript: all authors. Statistical analysis: Shah. Administrative/technical/ material support: Hemm, Coste, Lemaire, Schkommodau, Taub, Derost. Study supervision: Hemm, Lemaire, Taub. Supervision of PhD research: Hemm, Guzman, Taub, Schkommodau.

\section{Supplemental Information \\ Previous Presentations}

Portions of this work have been presented in poster form at the following conferences: 7th International IEEE Engineering in Medicine and Biology Society (EMBS) Neural Engineering Conference, Montpellier, France, April 22, 2015; Joint Annual Meeting of the Swiss Societies of Neuroradiology and Neurosurgery, Lucerne, Switzerland, September 10, 2015; 18th International Congress of Parkinson's Disease and Movement Disorders, Stockholm, Sweden, June 8, 2014; 20th Congress of the European Society for Stereotactic and Functional Neurosurgery, Lisbon, Portugal, September 26, 2012; and 46th Annual Conference of the German Society for Biomedical Engineering, Jena, Germany, September 16, 2012.

\section{Correspondence}

Simone Hemm, Institute of Medical and Analytical Technologies, University of Applied Sciences and Arts Northwestern Switzerland, Gruendenstrasse 40, Muttenz CH-4132, Switzerland. email: simone.hemm@fhnw.ch. 